\title{
Total PC Activity Is Increased in Uterine Lavage of Post-Menopausal Endometrial but Not Ovarian Cancer Patients
}

\author{
Sophea Heng 1,2,3, Andrew N. Stephens 2,4,6, Tom W. Jobling5,6 and Guiying Nie ${ }^{1,2,3 凶}$ \\ 1. Implantation and Placental Development Laboratory, Centre for Reproductive Health, Hudson Institute of Medical Research, Clayton, Victoria, 3168, \\ Australia; \\ 2. Department of Molecular and Translational Sciences, Monash University, Clayton, Victoria, 3800, Australia; \\ 3. Department of Biochemistry and Molecular Biology, Monash University, Clayton, Victoria, 3800, Australia; \\ 4. Centre for Cancer Research, Hudson Institute of Medical Research, Clayton, Victoria, 3168, Australia; \\ 5. Department of Obstetrics and Gynaecology, Monash University, Clayton, Victoria, Australia; \\ 6. Epworth Research Institute, Epworth HealthCare, Richmond, Victoria, Australia.
}

$\square$ Corresponding author: Guiying Nie, Hudson Institute of Medical Research, 27-31 Wright Street, Clayton, VIC 3168, Australia. Phone: +61 395944380 Fax: +61 395946125 Email: guiying.nie@hudson.org.au.

(C) Ivyspring International Publisher. Reproduction is permitted for personal, noncommercial use, provided that the article is in whole, unmodified, and properly cited. See http://ivyspring.com/terms for terms and conditions.

Received: 2016.05.30; Accepted: 2016.07.09; Published: 2016.08.12

\begin{abstract}
Endometrial and ovarian cancers are two most common cancers affecting women in their post-menopausal years. To date, there are no simple biochemical tests to detect these cancers at early stages. Our previous study has demonstrated that the activity of total proprotein convertases (PCs) is significantly increased in uterine lavage at all stages of endometrial cancer, suggesting uterine lavage which can be obtained relatively non-invasively may provide a simple tool for the detection of endometrial cancer. However, uterine lavage may also contain ovarian-derived factors, and PCs are also reported to be up-regulated in ovarian cancer. In this study we determined whether increases in uterine lavage PC activity are specific to endometrial cancer or are also associated with ovarian cancer. PC activity was detected in all uterine lavages examined but no difference was found between women with and without ovarian cancer. On the other hand, the PC activity was significantly higher in post-menopausal endometrial cancer patients, consistent with our previous report. These results suggest that measuring total PC activity in uterine lavage is a useful tool to detect endometrial cancer specifically.
\end{abstract}

Key words: proprotein convertase

\section{Introduction}

Ovarian and endometrial cancers are two most common gynecological malignancies [1], most often affecting women in their post-menopausal women years. However, recent studies have demonstrated that obesity is associated with increased incidence of endometrial cancer in peri-menopausal women [2]. Currently, there are no simple early detection methods available for both cancers, as most often these cancers are already well progressed before any symptoms are detected. In order to improve the survival rate, development of non-invasive detection tools is essential.
Proprotein convertases (PCs) are a family of serine proteases that share a consensus cleavage motif of $(K / R-(X) n-(K R) \downarrow(n=2,4$ or $6, X$ is any other amino acid) [3]. PCs are ubiquitously expressed in normal tissues, but also have a number of important functional roles in various cancers [4-6]. Our previous study has identified furin as the only PC member that is significantly up-regulated in post-menopausal endometrial cancer tissues [7]. In addition, the total PC activity in uterine lavage is significantly increased in endometrial cancer patients compared to controls [7]. These results suggest that measuring PC activity 
in uterine lavage may provide a non-invasive detection method for endometrial cancer [7].

However, PCs are also reported to be increased in ovarian cancer [8], and ovary-derived proteins are found in uterine lavages [9]. It is thus uncertain whether increases in lavage PC activity are specific to endometrial cancer or are also associated with ovarian cancer.

In this study, we determined whether the total PC activity in uterine lavage is increased in post-menopausal ovarian cancer patients, and whether assaying total PC activity in uterine lavage can distinguish endometrial and ovarian cancers.

\section{Materials and Methods}

\section{Collection of uterine lavages from ovarian and endometrial cancer patients}

Sample collection was approved by appropriate Institutional Ethics Committees, with written informed consent from individual patient. Uterine lavage was obtained as previously described [10]. In brief, $5 \mathrm{ml}$ of saline was infused transcervically into the uterine cavity with a fine catheter. The fluid was aspirated, centrifuged at $1000 \mathrm{~g}$ for $5 \mathrm{~min}$ to remove cellular debris, aliquoted and stored at $-80^{\circ} \mathrm{C}$. A total of 89 lavages samples were analyzed for this study, including post-menopausal non-cancer controls ( $\mathrm{n}=$ $8)$, ovarian cancer [benign tumors $(\mathrm{n}=21)$, borderline cancers $(\mathrm{n}=4)$, serous-papillary $(\mathrm{n}=21)$, serous peritoneal $(n=9)$, other serous subtypes $(n=8)$, other types of ovarian cancers, including clear cell ovarian tumors and malignant mixed mullerian tumors $($ MMMT) $(n=7)]$, and endometrial cancers $(n=11)$.

\section{Analysis of PC activity in uterine lavages}

Total PC activity was determined by the cleavage of a fluorogenic peptide substrate pERTKR-AMC (Bachem, Torrance, CA, USA) [10-12]. A final concentration of $100 \mu \mathrm{M}$ substrate was incubated with $50 \mu$ of each sample (neat) at $37^{\circ} \mathrm{C}$ in $1 / 2$ area 96-well plates. The real-time kinetics of substrate hydrolysis (release of AMC from the substrate) was monitored every $5 \mathrm{~min}$ for $60 \mathrm{~min}$ at excitation/emission of 355/460 nm (Wallac Victor 2 spectrophotometer, PerkinElmer, MA, USA), and the rate of substrate hydrolysis (AMC released per min) was calculated from the linear phase of each real-time kinetic progression curve. To confirm specificity of the assay, representative ovarian cancer lavages were assayed in the absence and presence of a potent PC inhibitor, decanoyl-Arg-Val-Lys-Arg-chloromethylketone (dec-RVKR-CMK, otherwise referred to as CMK) (Bachem, Bubendor, Switzerland) [7].

\section{Statistical analysis}

Data were expressed as mean \pm SEM. Statistical analysis used non-parametric, Mann Whitney and Kruskal Wallis test (PRISM, version 6.00, GraphPad software, San Diego, CA). ${ }^{* *} p<0.005$ was taken as significant.

\section{Results and Discussion}

Total PC activity was detected in all 89 lavage samples examined. The levels were not significantly different between non-cancer controls $(n=8)$, and ovarian benign tumors $(\mathrm{n}=21)$, borderline cancers ( $\mathrm{n}$ $=4)$, serous-papillary $(\mathrm{n}=21)$, serous peritoneal $(\mathrm{n}=$ $9)$, other serous subtypes $(n=8)$, or other types of ovarian cancer $(\mathrm{n}=7)$ (Figure 1A).

A

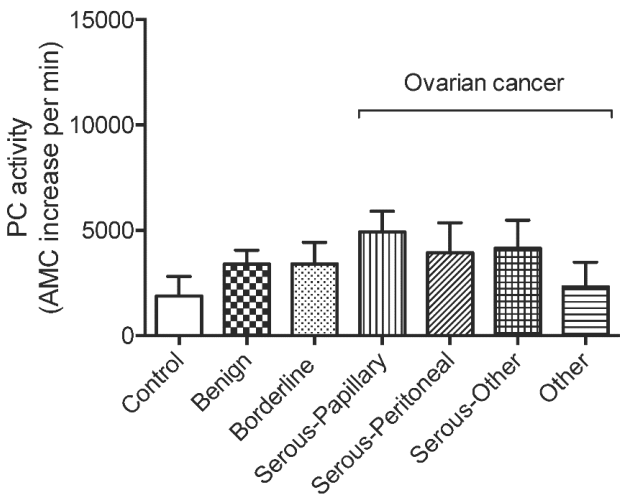

B
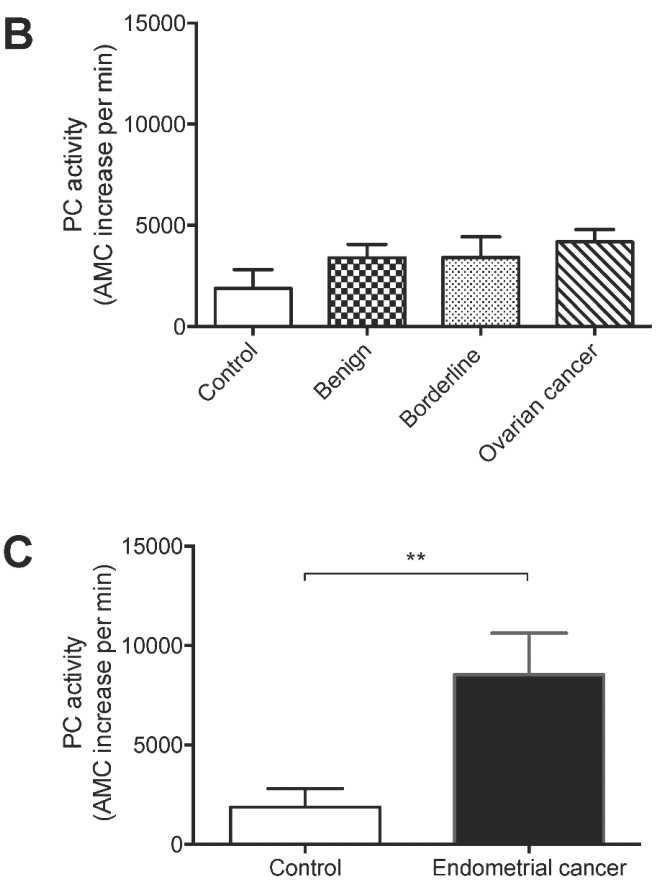

Figure 1: PC activity in uterine lavages from post-menopausal controls, ovarian cancer and endometrial cancer patients. (A) No significant differences were detected in ovarian cancer subtypes [serous-papillary $(n=21)$, serous peritoneal $(n=9)$, other serous subtypes $(n=8)$, other types of ovarian cancer $(n=7)$ ] compared to controls $(n=8)$, benign $(n=21)$ or borderline cancers $(n=4)$. (B) Comparison of all ovarian cancer subtypes as one group $(n=45)$ with controls $(n=8)$, benign tumors $(n=21)$, or borderline cancers $(n=4)$. (C) Total PC activity was significantly higher in endometrial cancer $(n=11)$ compared to controls $(n=8)$, ${ }^{*} p<0.005$. 
All ovarian cancer samples as one group $(n=45)$ were also not significantly different from controls, benign ovarian tumors $(n=21)$, or borderline ovarian cancers $(\mathrm{n}=4)$ (Figures 1A-B). On the other hand, total PC activity in endometrial cancer lavages $(n=11)$ was significantly higher than non-cancer controls ( $\mathrm{n}=$ 8) (Figure 1C), consistent with our previous findings [7].

To confirm PC specificity in uterine lavage from the ovarian cancer patients, representative ovarian cancer lavages were analyzed with and without a PC inhibitor dec-RVKR-CMK (Figure 2). The PC activity was completely abolished following the inhibitor treatment (Figure 2), confirming PC specificity.

These results suggest that although total PC activity is detected in uterine lavage of ovarian cancer patients, it is not significantly elevated than controls; this is in contrast to endometrial cancer, demonstrating the specificity of the assay to endometrial cancer [7].

In summary, only endometrial cancer but not ovarian patients showed significantly elevated PC activities in their uterine lavages. Therefore, total PC activity in uterine lavage can be used to detect endometrial cancer without being interfered by ovarian cancer.

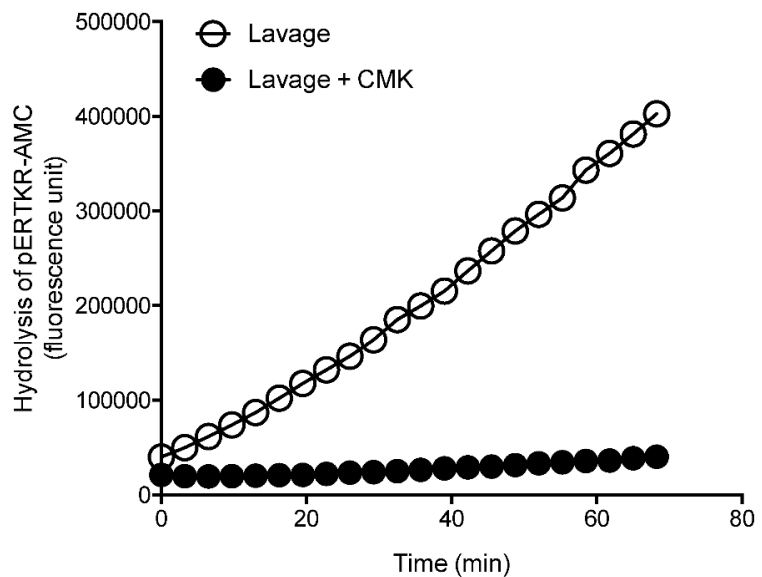

Figure 2: Inhibition of $\mathrm{PC}$ activity in uterine lavages from ovarian cancer patients by a potent PC inhibitor, decanoyl-Arg-Val-Lys-Arg-chloromethylketone (CMK). Representative progressive curves of PC activity with and without $50 \mu \mathrm{MCMK}$ in an ovarian cancer lavage is shown.

\section{Acknowledgements}

This work was supported by the National Health and Medical Research Council of Australia [Project grant \#1044182 and Fellowship \#1041835 (to GN)], the Ovarian Cancer Research Foundation, and the Victorian Government Operational Infrastructure Support Program. We thank research nurse Nicole Fairweather for collecting the clinical materials, and the patients who kindly donated uterine lavages for this study.

\section{Competing Interests}

The authors have declared that no competing interest exists.

\section{References}

1. Siegel RL, Miller KD, Jemal A. Cancer statistics, 2015. CA Cancer J Clin. 2015; 65: 5-29.

2. Gao Y, Dai X, Chen L, Lee AC, Tong M, Wise M, et al. Body Mass Index Is Positively Associated with Endometrial Cancer in Chinese Women, Especially Prior to Menopause. J Cancer. 2016; 7: 1169-73.

3. Seidah NG, Mayer G, Zaid A, Rousselet E, Nassoury N, Poirier S, et al. The activation and physiological functions of the proprotein convertases. Int J Biochem Cell Biol. 2008; 40: 1111-25

4. Bassi DE, Fu J, Lopez de Cicco R, Klein-Szanto AJ. Proprotein convertases: "master switches" in the regulation of tumor growth and progression. Mol Carcinog. 2005; 44: 151-61.

5. Bassi DE, Mahloogi H, Al-Saleem L, Lopez De Cicco R, Ridge JA, Klein-Szanto AJ. Elevated furin expression in aggressive human head and neck tumors and tumor cell lines. Mol Carcinog. 2001; 31: 224-32.

6. de Cicco RL, Bassi DE, Benavides F, Conti CJ, Klein-Szanto AJ. Inhibition of proprotein convertases: approaches to block squamous carcinoma development and progression. Mol Carcinog. 2007; 46: 654-9.

7. Singh H, Heng S, Nicholls PK, Li Y, Tai LT, Jobling T, et al. Proprotein convertases in post-menopausal endometrial cancer: distinctive regulation and non-invasive diagnosis. Biochemical and biophysical research communications. 2012; 419: 809-14.

8. Page RE, Klein-Szanto AJ, Litwin S, Nicolas E, Al-Jumaily R, Alexander P, et al. Increased expression of the pro-protein convertase furin predicts decreased survival in ovarian cancer. Cell Oncol. 2007; 29: 289-99.

9. Lopata A, Agresta F, Quinn MA, Smith C, Ostor AG, Salamonsen LA. Detection of endometrial cancer by determination of matrix metalloproteinases in the uterine cavity. Gynecologic oncology. 2003; 90: 318-24.

10. Heng S, Hannan NJ, Rombauts LJ, Salamonsen LA, Nie G. PC6 levels in uterine lavage are closely associated with uterine receptivity and significantly lower in a subgroup of women with unexplained infertility. Human reproduction. 2011; 26: 840-6.

11. Heng S, Cervero A, Simon C, Stephens AN, Li Y, Zhang J, et al. Proprotein convertase $5 / 6$ is critical for embryo implantation in women: regulating receptivity by cleaving EBP50, modulating ezrin binding, and membrane-cytoskeletal interactions. Endocrinology. 2011; 152: 5041-52.

12. Heng S, Dynon K, Li Y, Edgell T, Walton K, Rombauts LJ, et al. Development of a high-throughput assay for human proprotein convertase $5 / 6$ for detecting uterine receptivity. Anal Biochem. 2015; 475: 14-21. 